Onomástica Desde América Latina, n.2, v.1, julho - dezembro, 2020, p.122-143. ISSN 2675-2719

\title{
Sobre antroponimia en las calles de Santa Fe, Nuevo México ${ }^{1}$ About anthropnimy on the streets of Santa Fe, New Mexico
}

Miguel Reyes Contreras

Universidad Intercultural del Estado de México

https://orcid.org/0000-0003-3816-9189

reyescontramiguel@gmail.com

\section{Resumen}

La relación entre una calle y el antropónimo que la identifica va más allá de una mera forma de ubicación en un espacio geográfico. Implica una representación de la historia nacional, local, internacional y cultural. Al mismo tiempo es una especie de mecanismo de control por parte de los gobiernos pues sirven a lo administrativo, pero también se legitima la historia oficial en turno. El objetivo es analizar un corpus de 1260 nombres de calles de Santa Fe, Nuevo México los cuales contienen nombres personales para determinar la relación de estos nombres con la historia del país, del estado y de la ciudad. El panorama que ofrece el trabajo es que la ciudad de Santa Fe da la misma importancia a lo nacional como a lo local, lo cual permite una mayor creatividad en la nominación. A partir de este análisis también permite conocer la riqueza lingüística y cultural en la ciudad.

Palabras-clave: Odonimia; Antroponimia; Paisaje Lingüístico; Historia; Ciudad de Santa Fe, Nuevo México.

\begin{abstract}
The connection between a street and the anthroponym which identifies it goes beyond a simple way of locating in the geographical space. It constitutes a representation of the national, local, international and cultural history and, at the same time, it is a sort of government control since they not only serve administratively, but also legitimize the current official history. The aim of this paper is to analyze a corpus of 1260 street names of Santa Fe, New Mexico containing person names so the connection between these names with the national, state and local history can be established. The outcomes of this paper are that the city of Santa Fe considers equally important the national and the local affairs and people. This allows a greater creativity in the naming process. Hence, this analysis also permits the possibility to observe the linguistic and cultural richness in the city.
\end{abstract}

Keywords: Odonymy; Anthroponymy; Linguistic Landscape; History; City of Santa Fe; New Mexico.

\footnotetext{
${ }^{1}$ Producto derivado del Proyecto de Investigación: Onomástica y Lengua y Cultura como "Visiting profesorScholar in Residence" bajo el auspicio de la Comisión de Cooperación México-Estados Unidos (COMEXUS) y la beca Fulbright-García Robles (2018-2019) en el "Institute of American Indian Arts", Santa Fe, Nuevo México.
} 


\section{Introducción}

El presente trabajo se inscribe en los estudios de Paisaje Lingüístico (PL) dado que es parte de un proyecto más amplio, cuyo objetivo es el de describir el paisaje lingüístico (onomástico) de Nuevo México (NM) en los niveles toponímico (nombres de lugares), odonímico (nombres de calles) y apoteconímico (nombres de comercios), además de presentar la visibilidad de las lenguas, especialmente las indígenas, en el PL nuevo mexicano. Para los fines que a la antroponimia convienen, el presente es un trabajo combinado entre odonimia y antroponimia con una perspectiva histórica. Si las calles son una forma de representación de la historia oficial (NEETHLING, 2016: 146-148), este trabajo cumple el objetivo particular de analizar cómo se representa la historia nacional, estatal y local, además de otros aspectos a nivel internacional en las calles de Santa Fe, NM.

\section{Algunos aspectos conceptuales}

Ya sean personas, lugares, algunos objetos o animales, reales o ficticios, todos tienen un nombre y este no es gratuito, sino que obedece a ciertas reglas sociales y lingüísticas que varían por regiones y por tiempos. Esta serie de hechos respecto del nombre es estudiada por la Onomástica u Onomatología, es decir, la ciencia de los nombres. Los estudios onomásticos contribuyen, entre otros muchos aspectos, a la comprensión de los paisajes lingüísticos (por todos los espacios donde se usa la lengua escrita). Cuando se clasifican los objetos de estudio de la onomástica, encontramos tres grandes categorías: la toponomástica, la antroponomástica y la crematonimia, que estudian los nombres de lugar, de persona y de objetos, respectivamente (fig. 1). 


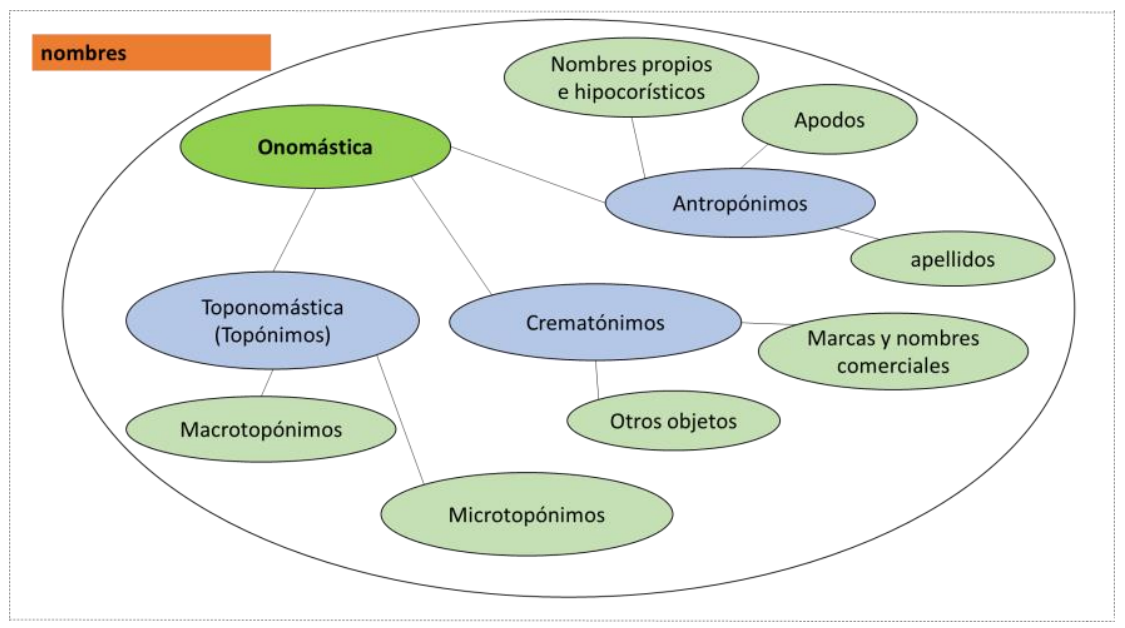

Figura 1. Los objetos de estudio de la onomástica (elaboración propia)

El Paisaje lingüístico es la forma en que se representa la lengua en los aparadores, en los signos comerciales, en posters, señalización oficial, señales de tráfico, etc., un tipo de estudio que amplía “...the range of sociolinguistic description from [...] speakers to spaces [], in which such speakers dwell, and in which they pick up and leave, so to speak, linguistics deposits, 'waste', signposts, roadmaps.” (BLOMMAERT, 2013: 1). Todo aspecto relacionado con el uso y representación del lenguaje en el espacio público es motivo de estudio en el Paisaje, que se clasifica de acuerdo con el objeto de estudio en paisaje cultural, lingüístico, artístico y, en este caso, onomástico. Hablar de paisaje onomástico (toponomástico y antroponomástico) es referirse al estudio tanto de los nombres de calles (llamado odonimia) como de los nombres de persona en estas calles (nombres per se, apellidos, hipocorísticos y apodos).

\section{Odonimia}

La odonimia, del griego odos "camino" y onoma "nombre" se refiere al estudio de las calles, plazas, carreteras y similares. Los temas que aborda esta subdisciplina incluyen conocer cómo se selecciona, cataloga, estandariza y se pone a disposición la información 
sobre la calle (NEETHLING, 2016: 144). Uno de los principales problemas a los que se enfrenta la odonimia es que la nomenclatura: carretera, calle, callejón, bulevar, paseo... etc. es confusa y no existen claras delimitaciones en cuanto al uso, puesto que depende de asuntos más políticos que terminológicos. Un ejemplo de ello es que, en Phoenix, Arizona, las calles corren de Este a Oeste y las avenidas de Norte a Sur ${ }^{2}$, lo que no ocurre en Santa Fe, donde pareciera que depende de la extensión y la función de la vía ${ }^{3}$, pero que en realidad no es funcional pues puede haber una calle más amplia que un bulevar o un camino más extenso que una avenida. Los gobiernos los consideran un instrumento de índole espacial pues tienen la función de orientación y como control administrativo, o sociales, pues conmemoran a personajes de la historia oficial en turno, por lo que es también un modo de producción simbólica del paisaje urbano y, por ende, es parte del paisaje cultural y lingüístico de un espacio geográfico determinado (NEETHLING, 2016).

La calle, como objeto de estudio ha sido visto desde varias perspectivas, como arena política (ADEBANWI, 2012; ALDERMAN, 2012, AZARYAHU, 2012), como un espacio para el análisis de la comunicación social (Cross, 2019), como una posibilidad de análisis lingüístico del léxico (JONES, 1970), como un espacio para la lectura del paisaje (FAIRCLOUGH, 1958; DÉSY, 1988; FORTIN, 2005; EBY, 2008; KALKMAN, 2019) o para estudiar la forma en que la identidad y la ideología se ven reflejadas en el lenguaje escrito de las calles (AZARYAHU, 2012; CABRERA, 2007; PINCHEVASKI, A. \& TORGOVNIK, 2003). Otros, desde la postura crítica, consideran a la calle como una especie de medallero político-social en el que se legitima la historia oficial (CAMACHO, 2014; CHALIER, 2014; GONZÁLEZ \& MURPHY, 1997; JÄRLEHE, 2015; MARGOTTI, 2018; MARTIN, 2013; O'NEILL, 1998; ORTIZ, 2009; POCCETTI, 2011).

\footnotetext{
${ }^{2}$ How streets, roads, and avenues are different https://www.youtube.com/watch?v=yqmso0c9CBs

${ }^{3}$ Datos proporcionados por el Dr. Carlos O. Ruiz, ingeniero de caminos de Nuevo México (entrevista: 20 de mayo de 2019)
} 


\section{Antroponimia}

Aunque el concepto de nombre propio demanda una discusión profunda sobre su situación teórica, en este apartado nos centraremos en definir de manera muy general cuatro conceptos clave de la antroponomástica, subdisciplina que comprende el estudio de los nombres de pila, apellidos, apodos e hipocorísticos y remitir al lector a revisar los trabajos de LOO LIU (2013), HOUGH (2014), KORTA (2014), LÓPEZ FRANCO (2014) y VAN LANGENDONCK (2007), entre otros para mayor detalle.

El concepto nombre propio representa un problema de orden terminológico pues para ello deben distinguirse entre nombres propios y nombres de pila. Estos se refieren a los nombres del santoral, por lo que automáticamente se excluyen los nombres que son invención de los padres (LÓPEZ FRANCO, 2012). VAN LANGENDONCK, (2007: 187) afirma que entre los nombres propios se incluyen "not only individual names (first names and most bynames 'nicknames') but also collective names, such as inhabitant names, certain bynames and especially family names", esto hace de la antroponimia un campo con un amplio espectro de investigación. Para los fines que a este trabajo competen, tomamos sólo los nombres personales.

El apellido, dice ZABALZA (2007: 259) es, a priori, el elemento que permite identificar a todo grupo familiar, asociado al sujeto social "casa", aunque no necesariamente el nombre de la casa es el apellido. El apellido "es el nombre antroponímico de la familia con que se distingue a las personas" (SHEMTOV-SHEMTOV, 2014: 5). FARKAS (2008: 366) explica que los apellidos también son "ethnic symbols, determined by their linguistic character and typical usage in the community", aunque hay varias tipologías. Por lo general, se clasifican en "a) patronymics, b) occupational names (and titles, dignities), c) nicknames (personal characteristics), and d) names referring to origin (toponymic or ethnic) (FARKAS, 2019: 29)" asociados, en ciertos casos con la casa o la familia. 
El apodo es, siguiendo a MOLINER (1998: 216) un "mote", un sobrenombre aplicado a veces a una persona, entre gente ordinaria, y muy frecuentemente en los pueblos, donde se transmite de padres a hijos; B, mote y el "apodo" es un sobrenombre, generalmente alusivo a alguna cualidad, semejanza de la persona a quien se aplica, por el que se conoce a esa persona”. Estos están asociados fuertemente con el apellido. El apodo es una traducción de nickname, "one of the most ancient anthroponymic units, the primary source of proper names and surnames" (GARAYEVAA, AKHMETZYANOVA AND KHISMATULLINAA, 2016: 1039). Molefe (1999: 3) retoma de Morgan (1971) que un "nickname is an eke-name, derived from the Old English verb can, meaning "to add to or augment". Thus, an eke-name was one given over and above the legal or baptismal name”. Es un término que también presenta problemas de delimitación terminológica pues:

Los apodos o motes son sobrenombres, así como los alias y pseudónimos, entre otros y se entienden como sobrenombres a los apelativos que sirven para volver a nombrar popularmente a personas que ya tienen el nombre oficial propio, el del Registro Civil, compuesto regularmente por su nombre y sus apellidos (RAMÍREZ, 2011: 558-559).

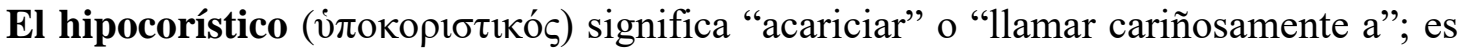
una palabra usada por la gramática para caracterizar los nombres que representan a otros nombres. Según FERNÁNDEZ Y PALENCIA (2015: 13) es “un tipo especial de nombres de pila que se usan en la lengua familiar como designaciones afectivas...", es decir, formas cariñosas usadas en el lenguaje familiar para los nombres propios que son de larga extensión y que, por su uso frecuente, se busca una forma más cómoda de mencionarlos abreviadamente. DO COUTO (2000: 50) lo denomina “o modo familiar, afetivo, de designarem as pessoas. Em alguns casos o nome hipocorístico é tirado do nome próprio da pessoa, freqüentemente (mas nem sempre) tomando-se-lhe a silaba tónica e reduplicando-a". 
A estas cuatro categorías agregamos el hagiónimo (nombre de un santo) y el caractónimo (nombre de un personaje literario o fílmico) (ICOS, 2019) ${ }^{4}$, que también se encuentran en el corpus.

\section{Metodología}

\section{El contexto de estudio}

Santa Fe, es la capital del Estado de Nuevo México. Se encuentra en la zona norte del Condado de Santa Fe. En la ciudad viven 65,000 habitantes. Desde su fundación, en 1609, como Real Villa de la Santa Fe de San Francisco de Asis, posee leyes estrictas para la planeación urbana, de entre las cuales destacan dos que competen a este trabajo: ningún edificio puede ser más alto que la catedral, por lo que la ciudad conserva un aspecto provinciano, y el hecho de que no se puede nombrar a una calle con un personaje vivo.

De manera global, el método de trabajo es Top-down, de lo general a lo particular, a través de la colecta en trabajo de campo, primero a nivel estado, luego a nivel condado, a nivel urbano y finalmente se delimitó un área especial de la ciudad, todo esto se complementó con entrevistas a algunos habitantes y con Carlos O. Ruiz, ingeniero de Caminos de Nuevo México, además de la investigación documental en bases de datos y material impreso. En este trabajo nos centramos en las calles del Condado de Santa Fe, primero recolectadas de la base de datos Streets of the World ${ }^{5}$ y posteriormente cotejadas con el Atlas de calles Street Maps Santa Fe County, Los Alamos, Española, Taos (HORTON: 2016). Los odónimos se clasificaron de la siguiente manera:

\footnotetext{
${ }^{4}$ En un sentido más amplio, también: "metaphor derived from the denotation of a common noun when transferred to a proper name" (Harder, 1980). La discusión en torno al tema la ofrecen BURELBACH (1983), CYPESS (1986), KALASHNIKOV (2013) y COATES (2018).

${ }^{5}$ www.streetsoftheworld.com
} 
1. Término genérico, el que aparece junto al nombre de la calle misma y la lengua en que se encuentra: calle, avenida, callejón, street, lane, road, strada, po, etc. Este aspecto de la odonimia no es de relevancia para los objetivos de este texto

2. Clasificación morfológica en simples, un solo componente sin contar el genérico, p. ej. Calle López, Sheridan Avenue, etc. y compuestos de dos (Adenois Lujan Road, Entrada Celedon y Nestora, etc,) y de tres elementos, los cuales contienen un elemento descriptivo respecto de la ubicación geográfica, p. ej. Traviesa de Camilo W(est) y Traviesa de Camilo East, ambos sin término genérico.

3. Categoría léxica: sustantivo, adjetivo, nombre, apellido y las lenguas de cada uno.

4. Notas sobre si los nombres pertenecen a un personaje histórico, religioso, local, del medio artístico, etc., además de datos enciclopédicos del personaje.

Una parte de los nombres propios en los odónimos para la clasificación se registraron por intuición, pero para comprobar la veracidad de los nombres se revisaron bases de datos como Behind the Name ${ }^{6}$ o búsquedas en línea. Además, para asegurar que en la ciudad existen portadores de los apellidos en nuestra base digital de datos, se consultaron la base de datos Names and Numbers ${ }^{7}$ y la versión impresa del directorio telefónico de Santa Fe.

\section{Sobre la antroponimia en las calles de Santa Fe}

En la recuperación global de datos para el proyecto general se recuperaron 3624 odónimos, de los cuales se extraen 1062, a los que denominamos odónimos antroponímicos: 840 simples y 222 compuestos (215 de dos componentes, 7 de 3). Cabe destacar que hallamos varios casos en los que el mismo término descriptivo se usa para describir varios genéricos, p. ej. Ortiz Lane, Ortiz Road, Ortiz Street, tanto en simples como en compuestos, razón por la cual el número es extenso. En cuanto a la relación onomástica con el nombre de las calles, en

\footnotetext{
${ }^{6}$ https://www.behindthename.com/

7 www.namesandnumbers.com/new-mexico/santa-fe/yellow-pages
} 
la fig. 2 se observa que, de este total de 1062, el nombre propio el apellido o la combinación de ambos en caso de compuestos, son las que más predominan, les sigue en frecuencia los hipocorísticos.

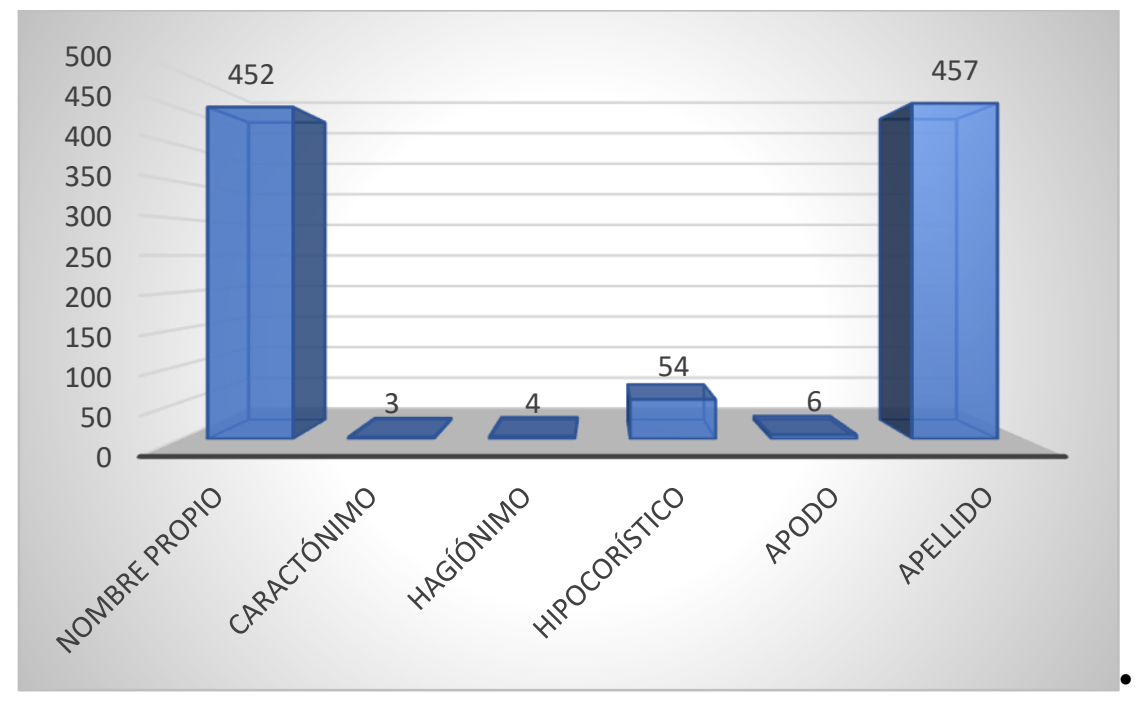

Fig. 2. Clasificación onomástica de los odónimos (elaboración propia).

\section{Anotaciones con respecto a la lengua}

La variedad de lenguas en las que se halla la nomenclatura de las calles de Santa Fe revela la fuerte raigambre histórica del pasado novohispano, además de la presencia de migración mexicana y la convivencia de hispanos, mexicanos, chicanos (esto sin considerar las otras nacionalidades). La nomenclatura es posible verla en cuatro lenguas: español, inglés, tewa e italiano en orden descendente. La idea de los pueblos de legitimar los terrenos de las reservas mostrando su lengua en espacios públicos se percibe en este caso por la destacable presencia del tewa (lengua del grupo indígena más numeroso en el condado). Es importante destacar que el español rebasa al inglés en la nomenclatura. Con respecto al elemento descriptor del odónimo, las lenguas representadas se presentan en la figura 3. De nueva cuenta el español sobresale casi un $30 \%$ por encima del inglés. Aunque de forma mínima, el tewa otra vez está presente. 


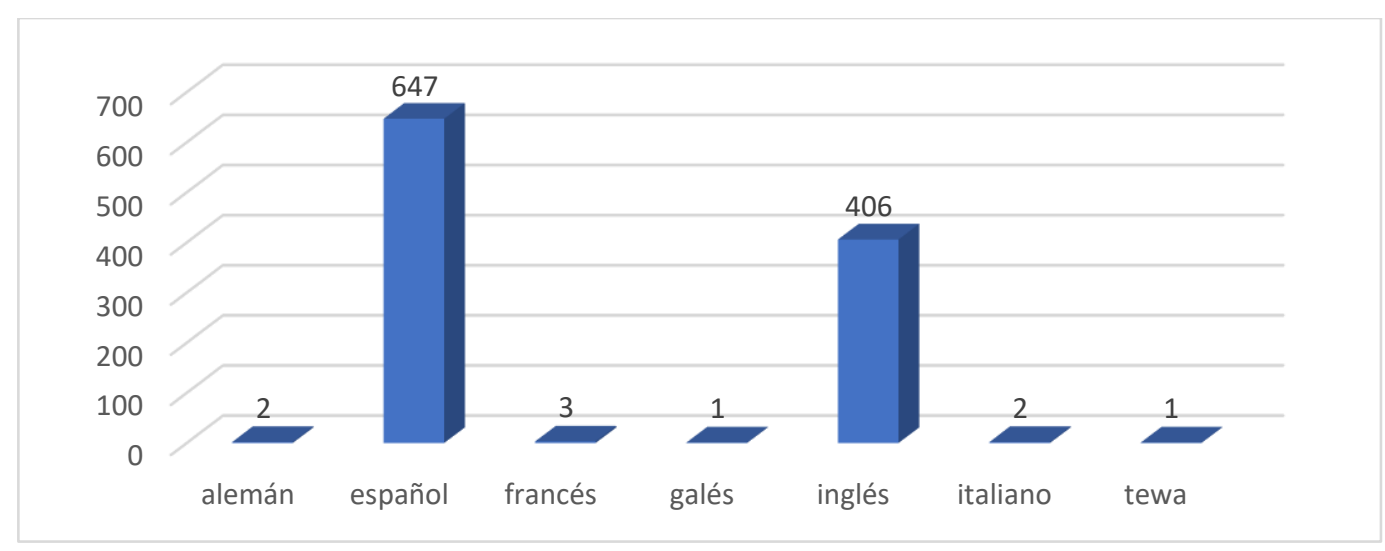

Fig. 3. Las lenguas de los términos descriptores en las calles de Santa Fe (elaboración propia)

\section{La historia en las calles}

La región que comprende el estado estuvo habitada por grupos Anasazi (que se disolvieron y se fusionaron para dar origen a los distintos "Pueblos" $"$ " de la actualidad, 19 en todo el estado), navajos, apaches, todos pertenecientes al grupo athabascos, además de hopis, de Arizona. Del total de antropónimos en el corpus, aparecen 50 personajes históricos y 31 que se han considerado personajes locales, pues, al hacer búsqueda de un apellido tanto en línea como en el directorio telefónico, nos remite a la calle misma donde viven personas con el mismo apellido. Presentaremos, a continuación, una serie de acontecimientos clave en la historia del Estado y del condado para conocer qué personajes han quedado registrados en la odonimia de Santa Fe.

Primeras exploraciones y conquistadores: Tras el descubrimiento de América por Cristóbal Colon, inician las exploraciones en el continente. El nombre de Colón permanece en una avenida que empieza en el Río Santa Fe y corre hacia el este, atravesando la avenida principal homónima de la otrora comunidad de Agua Fría.

\footnotetext{
${ }^{8}$ Se refiere a la forma en que se denominan actualmente: "Pueblo Indians" o Indios Pueblo y su adscripción es a la comunidad a la que pertenecen, Jemez, Cochiti, Zuni, Acoma, Laguna, Isleta, Sandia, Santa Ana, San Felipe, Zia, Santo Domingo, San Ildefonso, Tesuque, Nambe, Pojoaque, Santa Clara, Ohkay Owingeh, Picuris y Taos. (información proporcionada en el Hispanic Center, Albuquerque)
} 
Hacia 1521 empiezan las expediciones hacia el norte para expandir el terreno y uno de los primeros exploradores es Pedro de Alvarado y Contreras (Badajoz, 1485-Guadalajara, 1541), conquistador español que participó en la conquista de Cuba, en la exploración por Juan de Grijalva del golfo de México y de las costas de Yucatán, y en la conquista del Imperio azteca dirigida por Hernán Cortés, (conquistador de gran parte de América Central Guatemala, Honduras y El Salvador). Participó en la batalla sostenida contra los tlaxcaltecas dirigidos principalmente por Xicohténcatl. Una vez vencido este último, Tecuelhuetzin, su hija (bautizada como doña Luisa), tuvo un hijo con Alvarado (Pedro) y una hija, Leonor, quien se casó con Francisco de la Cueva, primo del duque de Alburquerque. El apellido familiar queda consignado en la Calle Alvarado.

Existen cuatro calles que llevan el nombre de Hernández de Córdoba (East y West Cordova Road, Cordova Lane y Cordova Place) (Córdoba, España, 1467-Sancti Spíritus, Cuba, 1517), el conquistador español que dirigió entre febrero y mayo de 1517 el denominado "descubrimiento de la Península de Yucatán". Otras tres calles con nombres de exploradores y conquistadores son Coronado Road (E y W) y Coronado Lane, en alusión a Francisco Vázquez de Coronado uno de los primeros exploradores y quien andaba en busca de la mítico reino de Cíbola. Este se hizo acompañar por dos personajes: el primero era Hernández Nuño Cabeza de Vaca, cuyo apellido se ve en Paseo C. De Baca, Ranchitos De Baca, Baca Lane, Baca Ranch Court, Baca Ranch Lane, Baca Ranch Loop, Baca Street, C De Baca Lane y Camino de Los Bacas. No obstante, es difícil deducir si se trata de él o bien de Ezequiel Cabeza de Baca, primer gobernador del estado debido a la variación ortográfica Vaca y Baca. El otro personaje es Juan de Oñate (Oñate Place y también Onate Place y Onate Street, quien pasa a la historia por tomar posesión en 1598, en nombre de Felipe II, de las tierras bañadas por el Río Grande (STEWART, 2008: 24-25). 


\section{La fundación de Santa Fe}

Se considera fundador de Santa Fe a Pedro de Peralta. En 1609 inicia la construcción de Santa Fe del Yunque, que después pasará a ser la Real Villa de la Santa Fe de San Francisco de Asis. Camino Peralta y Paseo De Peralta son las dos calles en las que queda consignado su nombre como personaje importante en la historia de la ciudad.

De 1680 a 1692 se levantan en armas los pueblos indígenas de la región (Pueblo Revolt) y es Don Diego de Vargas quien recupera los territorios tras haber sido desplazados durante 12 años hacia El Paso, en la frontera de Chihuahua y Texas. Los odónimos Vargas Court, E. y W. De Vargas Street, Corte Vargas, De Vargas, De Vargas Street, De Vargas Street $W$ y Don Diego Avenue $E$ y $W$ dan cuenta de la importancia de este personaje.

\section{La guerra de independencia y el siglo XIX}

A diferencia de muchas ciudades en México, donde es casi imprescindible que haya un odónimo "16 de septiembre", "Independencia" o "5 de mayo", en Santa Fe solo tienen tres personajes la Guerra de Independencia de EE. UU.: George Washington (Washington

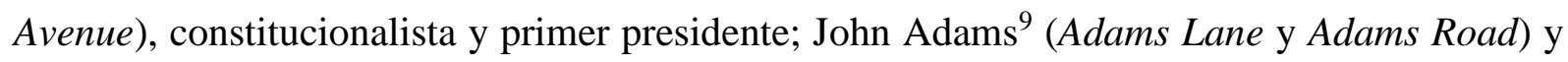
Benjamin Franklin, estadista, científico y constitucionalista (Franklin Avenue). Presidentes del siglo XIX son James Madison (Avenida Madison, Madison Road y Lugar de Madison), Zachary Taylor (Taylor Loop, Taylor Mountain ${ }^{10}$ y Taylor Road), Abraham Lincoln (Lincoln Avenue), Ulysses Grant (Grant Avenue y General Grant Avenue), James A. Garfield (Garfield

\footnotetext{
${ }^{9}$ (1735-1826), segundo presidente de los Estados Unidos. Está considerado como uno de los padres fundadores de esa patria. Ejerció como abogado, y desempeñó los cargos de delegado continental del Congreso y de vicepresidente, militando en el Partido Federalista de los Estados Unidos. (https://latam.historyplay.tv/hoy-en-lahistoria/nacio-john-adams-2do-presidente-de-los-eeuu)

${ }^{10}$ También hace referencia a un orónimo muy importante en el estado: Mount Taylor, nombrada por el teniente James H. Simpson en 1849 en honor al presidente Zachary Taylor, aunque Taylor Mountains es una serie montañosa nombrada por una familia local (JULYAN, 1998: 348).
} 
Street) y Grover Cleveland ${ }^{11}$ (Cleveland Road y Cleveland Street) y del siglo XX, solo tenemos a John F. Kennedy (Kennedy Court y Kennedy Road).

Como territorio (1846-1912) NM tuvo 19 representantes como gobernadores, de los cuales sólo el General Lew Wallace ${ }^{12}$ (General Wallace Drive) y Miguel A. Otero ${ }^{13}$ (Otero Street) aparecen en la nómina odonímica. En 1912, Nuevo México se convierte en el $47^{\circ}$ estado de los EE. UU. Su primer gobernador fue Ezequiel Cabeza de Baca; hay ocho odónimos con este apellido (véase la sección de conquistadores). Otros gobernadores son John E. Miles (Miles Lane, Governor Miles Road), John J. Dempsey (Governor Dempsey Street $^{14}$ ), Jerry Apodaca (Apodaca Hill Street), Toney Anaya (Calle Anaya $N$ y $S^{15}$ ), Gary Johnson (Johnson Lane, Johnson Mesa, Johnson Street, Johnsons Ranch Rd), Washington E. Lindsey (Lindsey Lane y Governor Lindsey Lane), Thomas J. Mabry (Governor Mabry Court) y Edwin L. Mechem (Governor Mechem Road).

\section{Personajes Locales}

Hay una cantidad considerable de personajes locales, sobre todo, en las calles de las comunidades pequeñas del condado. Personajes como Patrocino Romero Rd, Abel Griego Rd, Adenois Lujan Rd, Alvin Quintana Dr, Amado Romero Rd, Antonio Varela Rd, Arroyo Elfego Gomez, etc. portan apellidos locales, revisados en el directorio y otros medios, como los

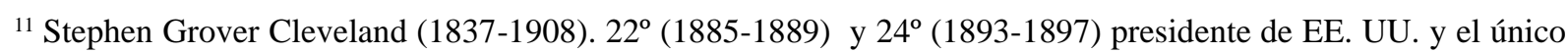
presidente de ese país en tener dos mandatos no consecutivos.

${ }^{12}$ Lewis Wallace (1827-1905). Abogado de la Unión general en guerra civil estadounidense, gobernador del Territorio de Nuevo México, político, diplomático y escritor de Indiana, Wallace es más conocido por su novela de carácter histórico Ben-Hur: A Tale of the Christ (1880). https://en.wikipedia.org/wiki/Lew_Wallace

${ }^{13}$ Miguel Antonio Otero II (1859-1944), apodado "Gillie" fue el 16o gobernador del Territorio de Nuevo México de 1897 a 1906 (también es el autor de varios libros sobre cultura del Este). https://en.wikipedia.org/wiki/Miguel_Antonio_Otero_(born_1859)

${ }^{14}$ Otro odónimo es General Dempsey Street en referencia a John Joseph Dempsey (1879-1958), Diputado de los EE. UU. y 13er gobernador de Nuevo México. https://en.wikipedia.org/wiki/John_J. Dempsey

${ }^{15}$ Otros odónimos probablemente en relación con este personaje son Anaya Ranch Road, B Anaya Road y Emma Anaya Lane
} 
Onomástica Desde América Latina, n.2, v.1, julho - dezembro, 2020, p.122-143. ISSN 2675-2719

obituarios $^{16}$. Una serie de odónimos con el apellido Ortiz, Ortiz Cr, Ortiz Ln, Ortiz Mountain Dr, Ortiz Ranch Rd, Ortiz Rd, Ortiz St, Rancho De Ortiz, Camino Luz Ortiz, Camino Ortiz, Casa De Ortiz o Edwardo Ortiz Dr refleja la interesante relación con la historia del condado. Por ejemplo, Ortiz Mountain Dr. remite a la orografía, pues una cadena de montañas (entre Golden y Madrid, NM) se nombraron en honor a José Francisco Ortiz, quien en 1828 descubrió oro en la zona y recibió el Ortiz Mine Grant en 1833 (JULYAN, 1998: 251-252).

El resto de los antropónimos es difícil de encasillarlos y clasificarlos si no es por medio de entrevistas a los habitantes de las calles, que no necesariamente conocen la historia de esta. Ejemplos como Andrews Rd, Andy ct, Andy's Park, Angie Ct, Anita Ln, Anita Pl, Anna Jean Ct, Anthony Ct, Antonio Ln, etc., que no nos permiten ver más allá del antropónimo que los nombra, pero son, en definitiva, apellidos locales.

Los hipocorísticos del corpus son 54 en primera y en 2a. posición, sólo 5. Entre los ejemplos, hallamos Aggie Dr., Alex St, Andy ct, Andy's Park, Angie Ct, Anita Ln, Anita Pl, Frankies Pl, Gregg Ave, Henry Ln, Jackie Rd, Jimmy Perez Rd. Los apodos son solo 6 (uno de ellos, compuesto). Los ejemplos son Camino Del Leo, Cuates Y Rabo, Don Tranquilo Dr, El Duane Ct, Rael Ln, y Rael Rd.

Nombres de personajes extranjeros son Hidalgo (uno de los condados fue nombrado en 1919 en honor al Tratado Guadalupe-Hidalgo, el cual toma su nombre de Miguel Hidalgo y Costilla $^{17}$ ), Churchill (Winston Churchill) y tal vez también la Calle Princesa Juana ${ }^{18}$. Hay antropónimos relacionados con el arte, como Puccini Plz (el compositor italiano), Pulitzer Trl

\footnotetext{
${ }^{16}$ https://www.geni.com/people/Adenois-Lujan/6000000003412543564

${ }^{17}$ Hidalgo County (Condado de Hidalgo) es el condado más al sur en Nuevo México. Éste fue nombrado en honor a la ciudad al norte de la Ciudad de México, donde se firmó el Tratado de Guadalupe Hidalgo, el cual se nombró en honor a Miguel Hidalgo y Costilla, el sacerdote conocido como "El padre de la Independencia de México". Este condado colinda con la frontera mexicana. También hay un condado en Texas con el mismo nombre https://www.hidalgocounty.us/
}

\footnotetext{
${ }^{18}$ Probablemente en alusión a Juana La Loca (o algún personaje local)
} 
Onomástica Desde América Latina, n.2, v.1, julho - dezembro, 2020, p.122-143. ISSN 2675-2719

(el periodista, fundador del prestigiado premio Pulitzer), Calle Lorca, Lorca Ct y Lorca Dr (el escritor), George Hayes Sr Pl (actor) ${ }^{19}$, Allan Hauser Ln (artista chiricahua), Ben Quintana (artista de Cochiti), Bob Haozous $R d$ (escultor Chiricahua) y Woo Peen $R d^{20}$ (artista plástico de NM). Agregamos también a la lista dos calles por su probable referencia a personajes famosos: Jose Alfredo Ln (quizá José Alfredo Jiménez, cantautor mexicano) y Camino Maria Feliz (sic) (pronunciado María Féliz, en alusión probable a la actriz mexicana María Félix).

Otros nombres que deben destacarse son los formados de manera creativa como Lois Ln (Lois Lane) cuya pronunciación nos remite a un personaje del cómic Superman, Lolita St, al personaje creado por Vladimir Nabokov y Penny Ln (Penny Lane) a una canción de The Beatles (1967). Cabe agregar Zepol Rd, cuya historia se debe a un conflicto entre dos grupos de la familia López, una de las cuales solo invirtió el apellido López para crear un odónimo nuevo sin perder el apellido: Zepol Rd. Finalmente, mencionaremos 3 caractónimos, que derivan de o se han convertido en nombres propios: Ben Hur Dr (Ben Hur, personaje de la novela homónima, es creación de Lewis Wallace, también gobernador del Estado), Calle Dulcinea y Don Quixote (sin nomenclatura), personajes de la famosa novela de Miguel de Cervantes Saavedra.

\section{Conclusiones}

Debido a que no hay una ley estricta ni comisión como en muchos países ${ }^{21}$, los residentes de la calle tienen derecho a nombrarla y no hay restricción para los nombres

\footnotetext{
${ }_{19}$ George "Gabby" Hayes fue un actor radiofónico, cinematográfico y televisivo estadounidense, conocido principalmente por sus numerosas actuaciones en películas de género western interpretando al personaje compañero del protagonista https://en.wikipedia.org/wiki/George \%22Gabby\%22_Hayes

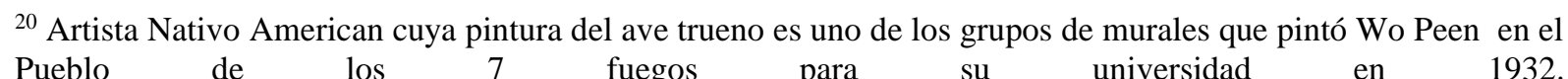
https://www.digitalcommonwealth.org/search/commonwealth-oai:r781x286v

${ }^{21}$ De la entrevista con Carlos O. Ruiz, Ingeniero de caminos de NM, 20 de mayo de 2019.
} 
(Quarrels End o Goa Way) $)^{22}$, lo que permite visibilizar mayor vitalidad lingüística en el paisaje onomástico de Santa Fe, al quedar en manos de los usuarios la nominación. Esto permite la creatividad nominativa y al ser una zona habitada tanto por descendientes de hispanos como por una gran población migrante se percibe una fuerte presencia del español, tanto en la nomenclatura de las calles como en los nombres de éstas. La presencia de lenguas indígenas también es destacable, como en el nombre del artista Woo Peen.

Sin embargo, más allá de encontrar la historia de los EE. UU. en las calles de Santa $\mathrm{Fe}$, lo que observamos es una riqueza cultural muy amplia. Está muy centrada en la historia local de la ciudad y de las pequeñas poblaciones, así como de los pueblos del condado. El corpus de odónimos recolectado no es sino una invitación a explorar la historia local y a analizar la riqueza cultural reflejada en los nombres de calle que no han optado por un antropónimo como descriptor. El recorrido por las calles es un recorrido por la historia. Los nombres de las calles nos revelan quién ha sido importante para la ciudad, quien merece ser recordado y quien no pudo ganarse un lugar, pero, sobre todo, revelan la dinámica social en la que se mueve un espacio urbano tan cosmopolita como Santa Fe.

Recebido em 19/04/2020

Aceito em 15/06/2020

Publicado em 29/07/2020

\section{Referencias}

Adebanwi, W. (2012) "Glocal naming and shaming: toponymic (inter) national relations on Lagos and New York's streets. African Affairs, Vol. 111, No. 445, 640-661. Disponible en https://www.jstor.org/stable/23357173 Accesado el 12-mayo-2019.

\footnotetext{
${ }^{22}$ Sobre todo, si la calle está en su propiedad (EDGE, 2017)
} 
Alderman, D. H. (2012) "Street Names as Memorial Arenas: The Reputational Politics of Commemorating Martin Luther King Jr. in a Georgia County”. En Historical Geography, Volume 30 (2002), 99-120.

Azaryahu, M. (2012) "Street Names and Political Identity: The Case of East Berlin”. Journal of Contemporary History, Vol. 21, No. 4, 581-604. Disponible en https://www.jstor.org/stable/260587, accesado el 12-mayo-2019.

Azaryahu, M. (2012). Renaming the past in post-Nazi Germany: Insights into the politics of street naming in Mannheim and Potsdam. En Cultural Geographies 19 (3), 385-400.

Behind the name (2019) https://www.behindthename.com/.

Blommaert, J. (2013) Ethnography, Superdiversity and Linguistic Landscapes. Chronicles of complexity. Bristol: Multilingual Matters.

Burelbach, F. M. (1983) “An Inquiry into Comic Making”. En Literary Onomastics Studies, Volume 10, 201-209.

Cabrera Silvera, E. (2017) "La memoria de una ciudad a través de las compositoras: un proyecto pedagógico en Santa Cruz de Tenerife". En XXII Coloquio de Historia Canarioamericana. Las Palmas de Gran Canaria., XXII-083, 1-9.

Camacho Guzmán, G. (2014) “Odonimia Herediana”. En Revista Káñina, vol. XXXVIII, 2014, 35-48.

Chalier, G. (2007) "Poder, historia y nominación: la toponimia urbana y la construcción de la identidad histórica”. En Mabel C. de Bulnes y José Marcilese (eds.). Cuestiones Políticas, socioculturales y económicas del sudoeste Bonaerense, actas de las IV Jornadas Interdisciplinarias del Sudoeste Bonaerense. Bahía Blanca: Universidad Nacional del Sur, 235-240. 
Onomástica Desde América Latina, n.2, v.1, julho - dezembro, 2020, p.122-143. ISSN 2675-2719

Coates, R. (2018). "Meaningfulness in literary naming within the framework of The Pragmatic Theory of Properhood (TPTP)”. En Onomastica Uralica 13, 191-196.

Cross, K. (2019) "The Uncanny Likeness of the Street: Visioning Community Through theLens of Social Media" in Pedram Dibazar, Judith Naeff Editor(s). Visualizing the Street. New Practices of Documenting, Navigating and Imagining the City. Amsterdam: Amsterdam University Press. Disponible en https://www.jstor.org/stable/j.ctv9hvqjh.13. Accesado el 13 de junio de 2019.

Cypess, S. M. (1986). Onomastics and Thematics in Balun-Canan. En Literary Onomastics $\begin{array}{lllll}\text { Studies, } & \text { Volume } & \text { 13, } & \text { 83-95. } & \text { Disponible }\end{array}$ http://digitalcommons.brockport.edu/los/vol13/iss1/8, consultado el 22 de junio de 2019.

Désy, C. (1988) Lecture du paysage par les noms de rues: exemples de Quebec. Montréal: Université McGill.

Do Couto, H. H. (2000) “Os hipocorísticos crioulos e o conceito de palavra ótima” en PAPIA, Vol. 10, pp. 50-65.

Eby, C. V. (2008) "The Street" as Humanitarian Narrative". En MELUS, Vol. 33, No. 1, Race, Space, and "National" Boundaries, 33-53. Disponible en https://www.jstor.org/stable/30029740, accesado el 12-mayo-2019.

Edge, S. (2017) “Quirky street names mirror county's unique history”. En Santa Fe New Mexican (May 2017). 6, Disponible https://www.santafenewmexican.com/news/local_news/quirky-street-names-mirrorcounty-s-unique-history/article_16781901-ea92-5c0f-a04e-e3aeab230849.html\#1 Consultado: 21 de abril de 2019 .

Fairclough, G. T. (1958). The Style of Street Names. En American Speech, Vol. 33, No. 4, 299-300. Disponible en https://www.jstor.org/stable/453876, accesado 12-mayo-2019 
Farkas, T. (2008). "Surnames of Foreign Origin in a Language Contact Situation. The Reasons and Ways of Their Changes and Their Influence on the Surname Stock in Hungary”. Proceeding of the $23^{\text {rd }}$ International Congress of Onomastic Sciences. York, 365-374.

Farkas, T. (2019). “A Surname Typology Project: The Lessons Learnt from the Distribution of the Most Frequent Hungarian Surnames." Onomastica Urálica. T. 13. HelsinkiDebrecen, 27-40.

Fernández, N. y Palencia, G. (2015) Análisis del fenómeno fonético del apócope en los hipocorísticos utilizados por los estudiantes de segundo año de Educación Media del Liceo Bolivariano Pedro Gual. Carabobo: Universidad de Carabobo. Tesis de Grado.

Fortin, J. (2005) «Dans le paysage Québécois : Napoléon, Joséphine et autres Bonaparte». En Cap-aux-Diamants, (81), 40-41.

Garayevaa, A. K., Akhmetzyanova, I. G. and Khismatullinaa, L. G. (2016). "The Significance of Learning Nicknames of Public Figures in Modern English and American Language Models of the World”. In International Journal of Environmental \& Science Education. Vol. 11, no. 4, 10337-10345. Disponible en https://files.eric.ed.gov/fulltext/EJ1119823.pdf Consultado el 27 de octubre de 2019.

González Faraco, J. C. \& Murphy, M. D. (1997) "Street Names and Political Regimes in an Andalusian Town”. En Ethnology, Vol. 36, No. 2, 123-148. Disponible en https://www.jstor.org/stable/3774079, accesado 12-mayo-2019.

Harder, K. B. (1980) "Onomastic Centrality," en Literary Onomastics Studies, Vol. 7, 33-54. Disponible en http://digitalcommons.brockport.edu/los/vol7/iss1/6. Accesado en mayo de 2019. 
Horton, J. (2016). Santa Fe County, Los Alamos, Española, Taos. Street maps. Albuquerque: Horton Family Maps.

Hough, C. (ed) (2014). The Oxford Handbook of Names and Naming. Oxford: Oxford University Press.

International Council of Onomastic Sciences, https://icosweb.net

Järlehe, J. (2015) "Genre and metacultural displays: the case of street-name signs" en Linguistic Landscape: An International Journal. Vol 3.3, 286-305.

Jones, O. F. (1970). “The use of Reykjavík street names”. En Scandinavian Studies, Vol. 42, No. 2, 190-196. Disponible en https://www.jstor.org/stable/40917060, accesado 12-mayo2019.

Julyan, R. (1998). The Place Names of New Mexico. Albuquerque: University of New Mexico Press.

Kalashnikov, A. (2013) "Rendering Onomastic Space in Don Quixote into English". En Els noms en la vida quotidiana. Actes del XXIV Congrés Internacional d'ICOS sobre Ciències Onomàstiques. Barcelona, 2249-2257.

Kalkman, S. (2019). "On or Beyond the Map? Google Maps and Street View in Rio de Janeiro's Favelas”. In Pedram Dibazar, Judith Naeff (Editors). Visualizing the Street. New Practices of Documenting, Navigating and Imagining the City. Amsterdam: Amsterdam University Press. Disponibel en https://www.jstor.org/stable/j.ctv9hvqjh.14.

Korta, K. (2014) “A simple theory of proper names”. En Revista Filosofía, Num. 53, 125-131.

Loo Liu, J. (2013) “The Two-Component Theory of Proper Names and Kripke's Puzzle”. En Abstracta, Volume 7, number 2, 19-39. 
López Franco, Y. G. (2012). Un siglo de nombres de pila en Tlalnepantla de Baz. Estudio lexicológico y sociolingüístico. México: UNAM-Plaza y Valdés.

López Franco, Y. G., (2014) "En torno al semantismo de los nombres propios. Entre debate y síntesis teórica". En Revista Trama, 10, núm. 20, 69-81.

Margotti, M. (2016) “Lo stradario del progresso. La laicizzazione degli spazi urbani nell'Italia post-unitaria. In HISTORIA RELIGIONUM, 8, pp. 55-66.

Martin, G. (2013). «Qui était Ernest Martin et pourquoi a-t-on nommé un chemin de Martston en son honneur? ». En Histoire Québec, 18(3), 22-24.

Molefe, L. (1999) Onomastic aspects of Zulu nicknames with special reference to source and functionality. Pretoria: University of South Africa. Tesis doctoral

Moliner, M. (2007) Diccionario del Uso del Español. Madrid: Gredos

Neethling, B. (2016). “Street Names: A Changing Urban Landscape” en Carole Hough (ed.). The Oxford Handbook of Names and Naming. Oxford: Oxford University Press., 144157.

O'Neill, C. E. (1998) “The French Regency and the Colonial Engineers: Street Names of Early New Orleans". en Louisiana History: The Journal of the Louisiana Historical Association, Vol. 39, No.2 (Spring, 1998), 207-214. Disponible en https://www.jstor.org/stable/4233494, accesado el 12-mayo-2019.

Ortiz, M. (2009) “El "panteón progresista” en las calles de Villa Mitre. Un caso peculiar de topografía urbana y construcción de la identidad”. En Memorias de III Jornadas Hum. H. A. Bahía Blanca: Universidad Nacional del Sur. 1-13. Disponible en www.jornadashumha.com.ar, consultado en octubre de 2018.

Pinchevaski, A. \& Torgovnik, E. (2003) "Signifying passages: the signs of change in Israeli street names". En Media, Culture \& Society, Vol. 24: 365-388. 
Poccetti, P. (2011). “L'Italia antica nell'odonimia della Roma di oggi” En Enzo Caffarelli e Massimo Fanfani (eds) Lo spettacolo delle parole. Studi di storia lingüística e di onomastica in ricordo di Sergio Raffaelli. Roma: SOCIETÀ EDITRICE ROMANA, 395417.

Ramírez Martínez, J., Ramírez García, R. y González Blanco, A. (2011). "Nopueser, Miama, El Hojalatero, Tecle: apodos de Nalda y otros pueblos de La Rioja" en En Els noms en la vida quotidiana. Actes del XXIV Congrés Internacional d'ICOS sobre Ciències Onomàstiques. Barcelona, 558-570.

Shemtov-Shemtov, S. (2014). Onomástica y el estudio de la etimología, transformación y morfología de los apellidos o nombres de las familias judías. Tel Aviv, BETH HATEFUTSOTH: THE MUSEUM OF THE JEWISH PEOPLE.

Stewart, G. R. (2008). Names on the Land. A historical account of place-naming in the United States. New York: The New York Review of Books.

Streets of the world (2018) Santa Fe Streets. Disponible en www.streetsoftheworld.com

Van Langendonck W. (2007). Theory and Typology of Proper Names. Berlin: Mouton de Gruyter.

Yellow pages. Santa Fe (2019) www.namesandnumbers.com/new-mexico/santa-fe/yellowpages

Zabalza Seguín, A. (2007). "Solar y apellido: la relación de la onomástica y la historia de la familia", en Societat d' Onomàstica: Butlletí interior, n. 104-105, pp.257-268. Disponible en http://dadun.unav.edu/handle/10171/28016 Consultado en septiembre de 2019. 